PROCEEDINGS OF THE

AMERICAN MATHEMATICAL SOCIETY

Volume 139, Number 4, April 2011, Pages 1455-1465

S 0002-9939(2010)10797-9

Article electronically published on November 18, 2010

\title{
VOLUME INTEGRAL MEANS OF HOLOMORPHIC FUNCTIONS
}

\author{
JIE XIAO AND KEHE ZHU
}

(Communicated by Richard Rochberg)

Abstract. The classical integral means of a holomorphic function $f$ in the unit disk are defined by

$$
\left[\frac{1}{2 \pi} \int_{0}^{2 \pi}\left|f\left(r e^{i \theta}\right)\right|^{p} d \theta\right]^{1 / p}, \quad 0 \leq r<1 .
$$

These integral means play an important role in modern complex analysis. In this note we consider integral means of holomorphic functions in the unit ball $\mathbb{B}_{n}$ in $\mathbb{C}^{n}$ with respect to weighted volume measures,

$$
M_{p, \alpha}(f, r)=\left[\frac{1}{v_{\alpha}\left(r \mathbb{B}_{n}\right)} \int_{r \mathbb{B}_{n}}|f(z)|^{p} d v_{\alpha}(z)\right]^{1 / p}, \quad 0 \leq r<1,
$$

where $\alpha$ is real, $d v_{\alpha}(z)=\left(1-|z|^{2}\right)^{\alpha} d v(z)$, and $d v$ is volume measure on $\mathbb{B}_{n}$. We show that $M_{p, \alpha}(f, r)$ increases with $r$ strictly unless $f$ is a constant, but in contrast with the classical case, $\log M_{p, \alpha}(f, r)$ is not always convex in $\log r$. As an application, we show that if $\alpha \leq-1, M_{p, \alpha}(f, r)$ is bounded in $r$ if and only if $f$ belongs to the Hardy space $H^{p}$, while if $\alpha>-1, M_{p, \alpha}(f, r)$ is bounded in $r$ if and only if $f$ is in the weighted Bergman space $A_{\alpha}^{p}$.

\section{INTRODUCTION}

Let $f$ be an analytic function $f$ on the unit disk $\mathbb{D}$ in the complex plane $\mathbb{C}$. For any $0<p<\infty$ the integral means of $f$ are defined by

$$
\mathrm{M}_{p}(f, r)=\left[\frac{1}{2 \pi} \int_{0}^{2 \pi}\left|f\left(r e^{i \theta}\right)\right|^{p} d \theta\right]^{1 / p}, \quad 0 \leq r<1 .
$$

The limit case $p=\infty$ takes the form

$$
\mathrm{M}_{\infty}(f, r)=\sup \{|f(z)|:|z|=r\}, \quad 0 \leq r<1 .
$$

The classical Hardy convexity theorem states that the function $\mathrm{M}_{p}(f, r)$ is increasing for $r \in[0,1)$ and the function $\log \mathrm{M}_{p}(f, r)$ is convex in $\log r$.

Hardy's convexity theorem is a fundamental result in modern complex and harmonic analysis in general and in the theory of Hardy spaces in particular. Immediate consequences of it include Hadamard's Three Lines Theorem and the justification

Received by the editors May 3, 2010.

2010 Mathematics Subject Classification. Primary 32A10, 32A36, 32A35, 51M25.

Key words and phrases. Volume/area integral means, monotonicity, logarithmic convexity, Bergman/Hardy spaces, isoperimetric-type inequalities, weighted Ricci curvatures.

The first author was supported in part by NSERC of Canada.

(C)2010 American Mathematical Society Reverts to public domain 28 years from publication 
for the identification of Hardy spaces $H^{p}$ in the unit disk with subspaces of $L^{p}$ on the unit circle.

In this paper we are going to consider an analogue of these integral means that are based on volume integrals. In fact, to go one step further, we will do this on the unit ball $\mathbb{B}_{n}$ in $\mathbb{C}^{n}$. Thus for any real number $\alpha$ we consider the weighted volume measure

$$
d v_{\alpha}(z)=\left(1-|z|^{2}\right)^{\alpha} d v(z)
$$

on $\mathbb{B}_{n}$, where $d v$ is volume measure on $\mathbb{B}_{n}$, normalized so that $v\left(\mathbb{B}_{n}\right)=1$.

If $f$ is holomorphic in $\mathbb{B}_{n}$ and $0<p<\infty$, we define

$$
M_{p, \alpha}(f, r)=\left[\frac{1}{v_{\alpha}\left(r \mathbb{B}_{n}\right)} \int_{r \mathbb{B}_{n}}|f(z)|^{p} d v_{\alpha}(z)\right]^{1 / p}, \quad 0 \leq r<1 .
$$

These will be called volume integral means of the function $f$. In the limit case $p=\infty$, we still define

$$
M_{\infty}(f, r)=\sup \left\{|f(z)|: z \in \mathbb{B}_{n},|z|=r\right\}, \quad 0 \leq r<1 .
$$

It follows from the maximum modulus principle that

$$
M_{p, \alpha}(f, r) \rightarrow M_{\infty}(f, r) \quad \text { as } \quad p \rightarrow \infty,
$$

regardless of the value of $\alpha$.

Recall that for $0<p<\infty$ the Hardy space $H^{p}$ of the unit ball consists of holomorphic functions $f$ in $\mathbb{B}_{n}$ such that

$$
\|f\|_{p}=: \sup \left\{M_{p}(f, r): 0 \leq r<1\right\}<\infty,
$$

where

$$
M_{p}(f, r)=\left[\int_{\mathbb{S}_{n}}|f(r \zeta)|^{p} d \sigma(\zeta)\right]^{1 / p}
$$

is the surface area integral mean of $f$, with $d \sigma$ being the normalized Lebesgue measure on the unit sphere $\mathbb{S}_{n}$ in $\mathbb{C}^{n}$.

It is well known that the measure $d v_{\alpha}$ is finite on $\mathbb{B}_{n}$ if and only if $\alpha>-1$. In what follows we are going to normalize the measure $d v_{\alpha}$ when $\alpha>-1$ by redefining

$$
d v_{\alpha}(z)=\kappa_{n, \alpha}\left(1-|z|^{2}\right)^{\alpha} d v(z)
$$

where

$$
\kappa_{n, \alpha}=\frac{\Gamma(n+\alpha+1)}{n ! \Gamma(\alpha+1)} .
$$

So $d v_{\alpha}$ becomes a probability measure in this case.

For $\alpha>-1$ and $0<p<\infty$ the weighted Bergman space $A_{\alpha}^{p}$ consists of holomorphic functions in $L^{p}\left(\mathbb{B}_{n}, d v_{\alpha}\right)$. If $H\left(\mathbb{B}_{n}\right)$ denotes the space of all holomorphic functions in $\mathbb{B}_{n}$, then

$$
A_{\alpha}^{p}=H\left(\mathbb{B}_{n}\right) \cap L^{p}\left(\mathbb{B}_{n}, d v_{\alpha}\right) .
$$

For any $f \in H\left(\mathbb{B}_{n}\right)$ we write

$$
\|f\|_{p, \alpha}=\left[\int_{\mathbb{B}_{n}}|f(z)|^{p} d v_{\alpha}(z)\right]^{1 / p} .
$$

Our main result is the following.

Theorem. Suppose $0<p<\infty, \alpha$ is real, and $f$ is holomorphic in $\mathbb{B}_{n}$.

(i) The function $r \mapsto M_{p, \alpha}(f, r)$ is strictly increasing on $[0,1)$ unless $f$ is constant. 
(ii) If $\alpha>-1$, then $M_{p, \alpha}(f, r)$ is bounded in $r$ if and only if $f$ is in the weighted Bergman space $A_{\alpha}^{p}$.

(iii) If $\alpha \leq-1$, then $M_{p, \alpha}(f, r)$ is bounded in $r$ if and only if $f$ belongs to the Hardy space $H^{p}$.

In the next section we prove the above Theorem and present several natural applications. In Section 3 we discuss the convexity of $\log M_{p, \alpha}(f, r)$ in $\log r$. We will show by an example that $\log M_{p, \alpha}(f, r)$ is not always convex in $\log r$. Furthermore, we conjecture that $\log M_{p, \alpha}(f, r)$ is convex in $\log r$ when $\alpha \leq 0$ and concave in $\log r$ when $\alpha>0$. Section 3 also contains some complex geometric evidence to support this conjecture.

\section{Monotonicity of $M_{p, \alpha}(f, r)$ And Applications}

We begin with the direct high-dimensional analogue of the classical integral means on the unit disk. The following result is probably known to experts, but we have been unable to find a reference. A proof is included here for completeness.

Lemma 1. Suppose $0<p \leq \infty$ and $f$ is a non-constant holomorphic function in $\mathbb{B}_{n}$. Then

(i) The function $M_{p}(f, r)$ is strictly increasing for $r$ over $[0,1)$.

(ii) The function $\log M_{p}(f, r)$ is convex in $\log r$.

Proof. It suffices to prove the result for $p<\infty$. The case $p=\infty$ will then follow from an obvious limit argument. So we assume $0<p<\infty$ for the rest of the proof.

Various proofs for the case $n=1$ can be found in [7, [10], and [18. The higherdimensional case follows from the rotation invariance of $d \sigma$ and the one-dimensional case for the so-called slice functions. In fact, we can write

$$
\begin{aligned}
M_{p}^{p}(f, r) & =\int_{\mathbb{S}_{n}}|f(r \zeta)|^{p} d \sigma(\zeta) \\
& =\frac{1}{2 \pi} \int_{0}^{2 \pi}\left(\int_{\mathbb{S}_{n}}\left|f\left(r \zeta e^{i \theta}\right)\right|^{p} d \sigma(\zeta)\right) d \theta \\
& =\int_{\mathbb{S}_{n}}\left(\frac{1}{2 \pi} \int_{0}^{2 \pi}\left|f_{\zeta}\left(r e^{i \theta}\right)\right|^{p} d \theta\right) d \sigma(\zeta),
\end{aligned}
$$

where $f_{\zeta}(w)=f(w \zeta)$ for $\zeta \in \mathbb{S}_{n}$ and $w \in \mathbb{D}$. If $f$ is not constant on $\mathbb{B}_{n}$, then the slice functions $f_{\zeta}$ will not be constant on $\mathbb{D}$ for $\zeta$ in the subset of $\mathbb{S}_{n}$ with positive measure. For each such $\zeta$, the function

$$
r \mapsto \frac{1}{2 \pi} \int_{0}^{2 \pi}\left|f_{\zeta}\left(r e^{i \theta}\right)\right|^{p} d \theta
$$

is strictly increasing on $[0,1)$. It follows that $M_{p}^{p}(f, r)$ is strictly increasing as a function of $r \in[0,1)$.

Alternatively, the monotonicity of $M_{p}(f, r)$ follows from the formula

$$
r \frac{d}{d r} M_{p}^{p}(f, r)=\frac{p^{2}}{2 n} \int_{r \mathbb{B}_{n}}|f(z)|^{p-2}|R f(z)|^{2}|z|^{-2 n} d v(z) .
$$

This formula was stated and proved in Theorem 4.20 of [22] under the assumption $f(0)=0$. But it can be shown that the assumption $f(0)=0$ is not necessary; see page 174 of [15]. 
To prove the logarithmic convexity of $M_{p}(f, r)$ in $\log r$, we follow the arguments used in Duren's book [7]. More specifically, according to Theorem 1.6 of [7, for any real number $\lambda$ the function

$$
\frac{r^{\lambda}}{2 \pi} \int_{0}^{2 \pi}\left|f_{\zeta}\left(r e^{i \theta}\right)\right|^{p} d \theta=\frac{1}{2 \pi} \int_{0}^{2 \pi}\left|r e^{i \theta}\right|^{\lambda}\left|f_{\zeta}\left(r e^{i \theta}\right)\right|^{p} d \theta
$$

is convex in $\log r$, as the function $|w|^{\lambda}\left|f_{\zeta}(w)\right|^{p}$ is subharmonic in $\mathbb{D}-\{0\}$. It is easy to see that integration with respect to $\zeta$ preserves the convexity in $\log r$. Thus $r^{\lambda} M_{p}^{p}(f, r)$ is convex in $\log r$ for any real $\lambda$. The convexity of $\log M_{p}(f, r)$ in $\log r$ then follows from exactly the same argument on page 10 of [7.

We are now ready to prove the first part of our main result. First observe that any normalizing constants used in the definition of $d v_{\alpha}$ will not make any difference in the definition of $M_{p, \alpha}(f, r)$, so we will not worry about these constants in the case $\alpha>-1$.

Theorem 2. Suppose $0<p<\infty, \alpha$ is real, and $f$ is holomorphic in $\mathbb{B}_{n}$. Then the function $r \mapsto M_{p, \alpha}(f, r)$ is strictly increasing on $[0,1)$ unless $f$ is constant.

Proof. According to integration in polar coordinates, we have

$$
\int_{r \mathbb{B}_{n}}|f(z)|^{p} d v_{\alpha}(z)=2 n \int_{0}^{r} \rho^{2 n-1}\left(1-\rho^{2}\right)^{\alpha} d \rho \int_{\mathbb{S}_{n}}|f(\rho \zeta)|^{p} d \sigma(\zeta) .
$$

It follows that

$$
\frac{d}{d r} \int_{r \mathbb{B}_{n}}|f(z)|^{p} d v_{\alpha}(z)=2 n r^{2 n-1}\left(1-r^{2}\right)^{\alpha} M_{p}^{p}(f, r) .
$$

Similarly, it follows from

$$
v_{\alpha}\left(r \mathbb{B}_{n}\right)=2 n \int_{0}^{r} \rho^{2 n-1}\left(1-\rho^{2}\right)^{\alpha} d \rho
$$

that

$$
\frac{d}{d r} v_{\alpha}\left(r \mathbb{B}_{n}\right)=2 n\left(1-r^{2}\right)^{\alpha} r^{2 n-1} .
$$

Integration by parts along with (11) and (2) then produces the following deficit formula between the two integral means:

$$
M_{p}^{p}(f, r)-M_{p, \alpha}^{p}(f, r)=\frac{1}{v_{\alpha}\left(r \mathbb{B}_{n}\right)} \int_{0}^{r}\left[\frac{d}{d t} M_{p}^{p}(f, t)\right] v_{\alpha}\left(t \mathbb{B}_{n}\right) d t .
$$

In particular, we see that

$$
\int_{r \mathbb{B}_{n}}|f(z)|^{p} d v_{\alpha}(z) \leq M_{p}^{p}(f, r) v_{\alpha}\left(r \mathbb{B}_{n}\right),
$$

where equality holds for some $r$ only when $f$ is constant.

We differentiate the function $M_{p, \alpha}^{p}(f, r)$ with respect to $r$ using the quotient rule and obtain

$$
\frac{d}{d r} M_{p, \alpha}^{p}(f, r)=\frac{v_{\alpha}\left(r \mathbb{B}_{n}\right) M_{p}^{p}(f, r)-\int_{r \mathbb{B}_{n}}|f(z)|^{p} d v_{\alpha}(z)}{\left(2 n r^{2 n-1}\left(1-r^{2}\right)^{\alpha}\right)^{-1} v_{\alpha}\left(r \mathbb{B}_{n}\right)^{2}} .
$$

Combining this with (4), we conclude that

$$
\frac{d}{d r} M_{p, \alpha}^{p}(f, r) \geq 0, \quad 0<r<1,
$$


where equality holds for some $r$ only when $f$ is constant. This shows that $M_{p, \alpha}^{p}(f, r)$, and hence $M_{p, \alpha}(f, r)$, is strictly increasing for $r \in[0,1)$ unless $f$ is constant.

The rest of this section is devoted to various natural applications of Theorem 2, including a proof of (ii)-(iii) of the main result (cf. Theorem 5 below). We begin with the easy observation that

$$
M_{p, \alpha}(f, 0)=\lim _{r \rightarrow 0^{+}} M_{p, \alpha}(f, r)=|f(0)| .
$$

The inequality

$$
|f(0)|<M_{p, \alpha}(f, r), \quad 0<r<1,
$$

where $f$ is non-constant, is a certain version of the mean value theorem. In particular, if $\alpha>-1$, then we can let $r \rightarrow 1$ in (5) to obtain $|f(0)|<\|f\|_{p, \alpha}$ whose limiting case $\alpha \rightarrow-1^{+}$appeared in 9 . Replacing $f$ by

$$
F(w)=f \circ \varphi_{z}(w)\left[\frac{1-|z|^{2}}{(1-\langle w, z\rangle)^{2}}\right]^{\frac{n+1+\alpha}{p}}
$$

leads to the following sharp pointwise estimate for functions in weighted Bergman spaces, which was first obtained in [19].

Corollary 3. Suppose $\alpha>-1, p>0$, and $f \in A_{\alpha}^{p}$. Then

$$
|f(z)| \leq \frac{\|f\|_{p, \alpha}}{\left(1-|z|^{2}\right)^{(n+\alpha+1) / p}}
$$

for all $z \in \mathbb{B}_{n}$. Moreover, equality holds at some point $a \in \mathbb{B}_{n}$ if and only if

$$
f(z)=c\left[\frac{1-|a|^{2}}{(1-\langle z, a\rangle)^{2}}\right]^{\frac{n+1+\alpha}{p}}
$$

for some constant $c$.

The limiting case $\alpha \rightarrow-1^{+}$of Corollary 3 is valid as well, provided we identify $A_{-1}^{p}$ with $H^{p}$. See Theorem 5 below and the remark preceding it.

Corollary 4. Suppose $\alpha \leq-1, p>0$, and $f$ is holomorphic in $\mathbb{B}_{n}$. Then the integral

$$
\int_{\mathbb{B}_{n}}|f(z)|^{p} d v_{\alpha}(z)=\sup _{r \in(0,1)} \int_{r \mathbb{B}_{n}}|f(z)|^{p} d v_{\alpha}(z)
$$

is finite if and only if $f$ is identically zero on $\mathbb{B}_{n}$.

Proof. For any $R \in(0,1)$ we know from Theorem 2 that

$$
M_{p, \alpha}^{p}(f, R) \leq \frac{\int_{r \mathbb{B}_{n}}|f(z)|^{p} d v_{\alpha}(z)}{v_{\alpha}\left(r \mathbb{B}_{n}\right)}, \quad R<r<1 .
$$

As $r \rightarrow 1^{-}$, we have $v_{\alpha}\left(r \mathbb{B}_{n}\right) \rightarrow+\infty$ and

$$
\int_{r \mathbb{B}_{n}}|f(z)|^{p} d v_{\alpha}(z) \rightarrow \int_{\mathbb{B}_{n}}|f(z)|^{p} d v_{\alpha}(z) .
$$

If the last integral above is finite, then we must have $M_{p, \alpha}(f, R)=0$ for each $R \in(0,1)$, which clearly implies that $f=0$. 
It is important to realize that the analyticity of $f$ is critical in the corollary above. For example, in the one-dimensional case, the result will be false if we just assume $f$ to be harmonic. In fact, the function

$$
f(z)=\left(1-|z|^{2}\right) /|1-z|^{2}
$$

is harmonic in the unit disk, and

$$
\int_{\mathbb{B}_{1}}|f(z)|^{p} d v_{-1}(z)<\infty
$$

as long as $p<1$. See 11 .

If $f$ is any holomorphic function in $\mathbb{B}_{n}$, then it follows from 23 , that

$$
\lim _{\alpha \rightarrow-1^{+}}\|f\|_{p, \alpha}=\|f\|_{p},, \quad 0<p<\infty .
$$

Thus we can think of the Hardy space $H^{p}$ as the limit case of the Bergman spaces $A_{\alpha}^{p}$ as $\alpha \rightarrow-1^{+}$. The following result makes this even more clear.

Theorem 5. Suppose $p>0$ and $f$ is holomorphic in $\mathbb{B}_{n}$.

(i) If $\alpha>-1$, then

$$
\sup \left\{M_{p, \alpha}(f, r): 0<r<1\right\}=\|f\|_{p, \alpha} .
$$

So in this case $f \in A_{\alpha}^{p}$ if and only if there is a positive constant $C$ such that $M_{p, \alpha}(f, r) \leq C$ for all $r \in[0,1)$.

(ii) If $\alpha \leq-1$, then

$$
\sup \left\{M_{p, \alpha}(f, r): 0<r<1\right\}=\|f\|_{p} .
$$

So in this case $f \in H^{p}$ if and only if there exists a positive constant $C$ such that $M_{p, \alpha}(f, r) \leq C$ for all $r \in[0,1)$.

Proof. When $\alpha>-1$, we have $v_{\alpha}\left(r \mathbb{B}_{n}\right) \rightarrow v_{\alpha}\left(\mathbb{B}_{n}\right)=1$ (according to our normalization) as $r \rightarrow 1^{-}$, and

$$
\lim _{r \rightarrow 1^{-}} \int_{r \mathbb{B}_{n}}|f(z)|^{p} d v_{\alpha}(z)=\int_{\mathbb{B}_{n}}|f(z)|^{p} d v_{\alpha}(z),
$$

whether the integral on the right hand side is finite or not. Thus (i) follows from Theorem 2 the monotonicity of $M_{p, \alpha}(f, r)$ in $r$.

When $\alpha \leq-1$ and $f$ is not identically zero, we have $v_{\alpha}\left(r \mathbb{B}_{n}\right) \rightarrow \infty$ as $r \rightarrow 1^{-}$, and according to Corollary 4 .

$$
\lim _{r \rightarrow 1^{-}} \int_{r \mathbb{B}_{n}}|f(z)|^{p} d v_{\alpha}(z)=\int_{\mathbb{B}_{n}}|f(z)|^{p} d v_{\alpha}(z)=\infty .
$$

Therefore, we can use the monotonicity of $M_{p, \alpha}(f, r)$ once again and apply L'Hôpital's rule to obtain (see the proof of Theorem 2 as well)

$$
\begin{aligned}
\sup _{0<r<1} M_{p, \alpha}^{p}(f, r) & =\lim _{r \rightarrow 1^{-}} M_{p, \alpha}^{p}(f, r) \\
& =\lim _{r \rightarrow 1^{-}} \frac{\int_{r \mathbb{B}_{n}}|f(z)|^{p}\left(1-|z|^{2}\right)^{\alpha} d v(z)}{\int_{r \mathbb{B}_{n}}\left(1-|z|^{2}\right)^{\alpha} d v(z)} \\
& =\lim _{r \rightarrow 1^{-}} \frac{2 n r^{2 n-1}\left(1-r^{2}\right)^{\alpha} \int_{\mathbb{S}_{n}}|f(r \zeta)|^{p} d \sigma(\zeta)}{2 n r^{2 n-1}\left(1-r^{2}\right)^{\alpha}} \\
& =\lim _{r \rightarrow 1^{-}} M_{p}^{p}(f, r)=\|f\|_{p}^{p} .
\end{aligned}
$$


This proves part (ii).

The following estimate is clearly a direct consequence of Theorem 2 .

Corollary 6. Suppose $\alpha>-1,0<p<\infty$, and $f$ is in $A_{\alpha}^{p}$. Then

$$
\int_{r \mathbb{B}_{n}}|f(z)|^{p} d v_{\alpha}(z) \leq v_{\alpha}\left(r \mathbb{B}_{n}\right) \int_{\mathbb{B}_{n}}|f(z)|^{p} d v_{\alpha}(z)
$$

for all $r \in[0,1]$. Moreover, equality holds for some $r \in[0,1)$ only when $f$ is constant.

This, in turn, yields another monotonicity principle as follows.

Corollary 7. Suppose $f$ is a non-constant holomorphic function in $\mathbb{B}_{n}$. Then

(i) The function $p \mapsto\|f\|_{p, \alpha}$ is strictly increasing for $p \in(0, \infty)$.

(ii) The function $\alpha \mapsto\|f\|_{p, \alpha}$ is strictly decreasing for $\alpha \in(-1, \infty)$.

Proof. Part (i) follows easily from Hölder's inequality and its equality case. So the non-trivial part is (ii).

Bringing the normalization constant back, we recall from the proof of Theorem 2 that

$$
\frac{d}{d r} \int_{r \mathbb{B}_{n}}|f|^{p} d v_{\alpha}=2 n \kappa_{n, \alpha}\left(1-r^{2}\right)^{\alpha} r^{2 n-1} \int_{\mathbb{S}_{n}}|f(r \zeta)|^{p} d \sigma(\zeta)
$$

We integrate by parts and use Corollary [6 to obtain that if $-1<\alpha_{1}<\alpha_{2}<\infty$ and $\|f\|_{p, \alpha_{1}}<\infty$, then

$$
\begin{aligned}
\|f\|_{p, \alpha_{2}}^{p}= & \int_{0}^{1} \frac{d}{d r}\left(\int_{r \mathbb{B}_{n}}|f|^{p} d v_{\alpha_{2}}\right) d r \\
= & \frac{\kappa_{n, \alpha_{2}}}{\kappa_{n, \alpha_{1}}} \int_{0}^{1}\left(1-r^{2}\right)^{\alpha_{2}-\alpha_{1}} \frac{d}{d r}\left(\int_{r \mathbb{B}_{n}}|f|^{p} d v_{\alpha_{1}}\right) d r \\
= & \frac{\kappa_{n, \alpha_{2}}}{\kappa_{n, \alpha_{1}}}\left[\left.\left(1-r^{2}\right)^{\alpha_{2}-\alpha_{1}} \int_{r \mathbb{B}_{n}}|f|^{p} d v_{\alpha_{1}}\right|_{0} ^{1}\right. \\
& \left.-\int_{0}^{1}\left(\int_{r \mathbb{B}_{n}}|f|^{p} d v_{\alpha_{1}}\right) d\left(1-r^{2}\right)^{\alpha_{2}-\alpha_{1}}\right] \\
= & -\frac{\kappa_{n, \alpha_{2}}}{\kappa_{n, \alpha_{1}}} \int_{0}^{1}\left(\int_{r \mathbb{B}_{n}}|f|^{p} d v_{\alpha_{1}}\right) d\left(1-r^{2}\right)^{\alpha_{2}-\alpha_{1}} \\
\leq & -\frac{\kappa_{n, \alpha_{2}}}{\kappa_{n, \alpha_{1}}} \int_{0}^{1}\left(v_{\alpha_{1}}\left(r \mathbb{B}_{n}\right)\|f\|_{p, \alpha_{1}}^{p}\right) d\left(1-r^{2}\right)^{\alpha_{2}-\alpha_{1}} \\
= & \|f\|_{p, \alpha_{1}}^{p} .
\end{aligned}
$$

This proves the monotonicity $\|f\|_{p, \alpha_{2}} \leq\|f\|_{p, \alpha_{1}}$. If equality occurs for some $\alpha_{1}$ and $\alpha_{2}$, then the previous estimates would force

$$
\int_{r \mathbb{B}_{n}}|f(z)|^{p} d v_{\alpha_{1}}(z)=v_{\alpha_{1}}\left(r \mathbb{B}_{n}\right)\|f\|_{p, \alpha_{1}}^{p},
$$

which, according to Corollary [6, implies that $f$ must be a constant.

The results in Corollary 7 above are optimal embeddings of weighted Bergman spaces. The following corollary gives several more such embeddings as well as some isoperimetric-type inequalities. 
Corollary 8. Suppose $p>0, \alpha>-1, \epsilon \geq p(\alpha+1) / n$, and $f$ is holomorphic in $\mathbb{B}_{n}$.

(i) We always have $\|f\|_{p, \alpha} \leq\|f\|_{p}$. Equality occurs if and only if $f$ is a constant, provided the quantities are finite.

(ii) We always have $\|f\|_{p(n+1+\alpha) / n, \alpha} \leq\|f\|_{p+\epsilon}$. Equality occurs if and only if $f$ is a constant, provided the quantities are finite.

(iii) If $f$ is a zero-free function in $H^{p}$ and $m$ is a natural number, then we always have $\|f\|_{p m,(m-1) n-1} \leq\|f\|_{p}$. Equality occurs if and only if $f(z)=$ $c(1-\langle z, w\rangle)^{-2 n / p}$, where $c \in \mathbb{C}$ and $w \in \mathbb{B}_{n}$.

Proof. The inequality in part (i) is obvious. If it becomes equality and the norms are finite, then for any $\beta \in(-1, \alpha)$ we use Corollary $[6$ to get

$$
\|f\|_{p} \geq\|f\|_{p, \beta} \geq\|f\|_{p, \alpha}
$$

which implies that $\|f\|_{p, \beta}=\|f\|_{p, \alpha}$, and so $f$ is constant.

To prove part (ii), write $q=p(n+1+\alpha) / n$. From (i) and Hölder's inequality it follows that

$$
\|f\|_{q, \alpha} \leq\|f\|_{q} \leq\|f\|_{p+\epsilon}
$$

with equalities if and only if $f$ is a constant, provided the norms are finite.

Part (iii) follows from [4]. In fact, according to Theorem 5.1 in [4, if $g$ is in $H^{2}$, then $g^{m}$ belongs to $A_{(m-1) n-1}^{2}$ and

$$
\left\|g^{m}\right\|_{2,(m-1) n-1}^{\frac{1}{m}} \leq\|g\|_{2},
$$

with equality occuring when and only when $g(z)=c(1-\langle z, w\rangle)^{-n}$ for some constant $c$ and some point $w \in \mathbb{B}_{n}$. Since $f$ is zero-free, taking $g=f^{\frac{p}{2}}$ in the last inequality yields

$$
\|f\|_{m p,(m-1) n-1} \leq\|f\|_{p}
$$

with equality occuring if and only if $f(z)=c(1-\langle z, w\rangle)^{-2 n / p}$ for some constant $c$ and some point $w \in \mathbb{B}_{n}$.

When $n=1$, part (iii) above is related to [17, page 97, Theorem 19.9], 12] and [20], which extended Carleman's argument for the isoperimetric inequality

$$
\frac{\text { Area }}{\pi} \leq\left(\frac{\text { Length }}{2 \pi}\right)^{2}
$$

on minimal surfaces $[6$.

For our final application in this section we specialize to the unit open disk $\mathbb{D}$ equipped with the normalized area measure $d A$. This will enable us to see the geometry much more clearly.

Corollary 9. If $f$ is analytic in the unit disk, then

$$
\int_{r \mathbb{D}}\left|f^{\prime}(z)\right|^{2} d A(z) \leq r^{2} \int_{\mathbb{D}}\left|f^{\prime}(z)\right|^{2} d A(z)
$$

for all $0 \leq r<1$. Moreover, equality holds for some $r \in[0,1)$ if and only if $f(z)=a z+b$ is a linear function.

This result is almost trivial in terms of Taylor expansions, and it is clearly a special case of Corollary 6. If $f$ is univalent, the Dirichlet integrals

$$
\int_{r \mathbb{D}}\left|f^{\prime}(z)\right|^{2} d A(z), \quad \int_{\mathbb{D}}\left|f^{\prime}(z)\right|^{2} d A(z)
$$


represent the areas of the images of $r \mathbb{D}$ and $\mathbb{D}$ under the mapping $f$, and the inequalities in Corollary 9 can be rephrased as

$$
A(f(r \mathbb{D})) \leq r^{2} A(f(\mathbb{D})), \quad 0 \leq r<1,
$$

where $A(\Omega)$ denotes the area of $\Omega$ in the complex plane. This inequality was obtained and discussed in [5], and there it was called the Area Schwarz Lemma. When $f$ is not necessarily univalent, the above Dirichlet integrals represent the areas of the images of $r \mathbb{D}$ and $\mathbb{D}$ under $f$ when multiplicities are taken into consideration. Alternatively, these integrals represent the areas of Riemann surfaces $(r \mathbb{D}, f)$ and $(\mathbb{D}, f)$. Thus Corollary 9 can be considered a certain version of the Schwarz Lemma for Riemann surfaces (cf. [14, 1] and [21]).

\section{LOGARITHMiC CONVEXITY}

In light of Lemma 1(ii) we naturally ask if the volume integral means $M_{p, \alpha}(f, r)$ are also logarithmically convex in $\log r$. Unlike the monotonicity issue, the logarithmic convexity problem is more delicate and the answer depends on the weight parameter $\alpha$. The following example shows that $\log M_{p, \alpha}(f, r)$ could be convex in $\log r$ for some $\alpha$, but it could also be concave for some other $\alpha$.

Example 10. Let $n=1$ and $f$ be holomorphic in the unit disk $\mathbb{D}$.

(i) If $1 \leq p<\infty$ and $\alpha \in\{-1,0\}$, then $\log M_{p, \alpha}(f, r)$ is a convex function of $\log r$.

(ii) If $p=2, \alpha=1$, and $f(z)=z$ on $\mathbb{D}$, then $\log M_{p, \alpha}(f, r)$ is a concave function of $\log r$.

Proof. Note that

$$
M_{p, 0}(f, r)=\left[\int_{\mathbb{D}}|f(r z)|^{p} d A(z)\right]^{1 / p} .
$$

It follows from Taylor's Banach space method in [18, Theorem 3.3] that $\log M_{p, 0}(f, r)$ is a convex function of $\log r$. The case when $\alpha=-1$ is the classical Hardy convexity theorem. This proves part (i).

To prove part (ii), we recall that

$$
d A_{1}(z)=2\left(1-|z|^{2}\right) d A(z), \quad z \in \mathbb{D},
$$

and

as well as

$$
A_{1}(r \mathbb{D})=2 \int_{r \mathbb{D}}\left(1-|z|^{2}\right) d A(z)=r^{2}\left(2-r^{2}\right)
$$

$$
\int_{r \mathbb{D}}|f(z)|^{2} d A_{1}(z)=2 \int_{r \mathbb{D}}|z|^{2}\left(1-|z|^{2}\right) d A(z)=r^{4}\left(1-2 r^{2} / 3\right) .
$$

Write

$$
F=M_{2,1}^{2}(z, r)=\frac{r^{4}\left(1-2 r^{2} / 3\right)}{r^{2}\left(2-r^{2}\right)}=\frac{1-2 r^{2} / 3}{2-r^{2}} r^{2}, \quad 0 \leq r<1 .
$$

If we set $t=\log r$, then $-\infty<t<0$ and

$$
F=\frac{1-2 e^{2 t} / 3}{2-e^{2 t}} e^{2 t}
$$

It follows that

$$
\log F=x+\log \left(1-2 e^{x} / 3\right)-\log \left(2-e^{x}\right), \quad x=2 t .
$$


Elementary calculations show that

$$
\frac{d^{2}}{d x^{2}} \log F=\frac{2 e^{x}}{3\left(2-e^{x}\right)^{2}\left(1-2 e^{x} / 3\right)^{2}}\left[\frac{1}{3} e^{2 x}-1\right] .
$$

Since $-\infty<x<0$, we must have

$$
\frac{d^{2}}{d x^{2}} \log F<0, \quad-\infty<x<0 .
$$

This shows that $\log M_{2,1}(z, r)$ is concave in $\log r$.

To better understand the dependence of logarithmic convexity on the weight parameter, let us take a closer look at the problem from a complex geometric viewpoint. According to [2], [16] and [13, a weighted Ricci curvature tensor on $\mathbb{B}_{n}$ is defined by

$$
\operatorname{Ric}_{\alpha}:=\operatorname{Ric}_{0}-\text { Hess } \log \left(1-|z|^{2}\right)^{\alpha},
$$

where $\mathrm{Ric}_{0}$ vanishes as the Ricci curvature tensor of the original ball $\left(\mathbb{B}_{n}, v\right)$, and

$$
\text { Hess } \log \left(1-|z|^{2}\right)^{\alpha}=\left(\frac{\partial^{2} \log \left(1-|z|^{2}\right)^{\alpha}}{\partial z_{k} \partial \bar{z}_{l}}\right)_{1 \leq k, l \leq n}
$$

is the complex Hessian matrix of $\log \left(1-|z|^{2}\right)^{\alpha}$. Recall that the Bergman kernel of $\mathbb{B}_{n}$ is given by

$$
K(z, w)=\frac{1}{(1-\langle z, w\rangle)^{n+1}}, \quad z, w \in \mathbb{B}_{n} .
$$

We follow [3] and [8] to write the Bergman metric as

$$
d s_{B}^{2}=\sum_{k, l=1}^{n} g_{k \bar{l}} d z_{k} d \bar{z}_{l}, \quad \text { where } \quad g_{k \bar{l}}=\frac{\partial^{2} K(z, z)}{\partial z_{k} \partial \bar{z}_{l}},
$$

and to write the Ricci curvature tensor of $d s_{B}^{2}$ as

$$
\left(\operatorname{Ric}_{k \bar{l}}\right):=\left(-\frac{\partial^{2} \operatorname{det}\left(g_{k \bar{l}}\right)}{\partial z_{k} \partial \bar{z}_{l}}\right) \text {. }
$$

A direct computation (see pages 22-23 of 22] for example) shows that

$$
\operatorname{Ric}_{\alpha}=-\frac{\alpha}{n+1}\left(\operatorname{Ric}_{k \bar{l}}\right)=\frac{\alpha}{n+1}\left(g_{k \bar{l}}\right) .
$$

Consequently, the sign of $\alpha$ completely determines the sign of $\mathrm{Ric}_{\alpha}$. In particular, when $\alpha \in(-\infty, 0)$, the weighted complex ball $\left(\mathbb{B}_{n}, v_{\alpha}\right)$ can be viewed as a KählerEinsten manifold with negative weighted Ricci curvature.

It seems reasonable to expect that the weighted Ricci curvature is somehow related to the volume integral means of holomorphic functions, especially when convexity issues are concerned. Example 10 appears to support this intuition, and we are tempted to make the following conjecture.

Conjecture 11. Suppose $0<p<\infty, \alpha$ is real, and $f$ is a non-constant holomorphic function on $\mathbb{B}_{n}$. Then the function $r \mapsto \log M_{p, \alpha}(f, r)$ is convex in $\log r$ when $\alpha \leq 0$ and concave in $\log r$ when $\alpha>0$. Furthermore, the function

$$
\frac{d}{d r}\left(r \frac{d}{d r} \log M_{p, \alpha}(f, r)\right)=\frac{d}{d r} \log M_{p, \alpha}(f, r)+r \frac{d^{2}}{d r^{2}} \log M_{p, \alpha}(f, r)
$$


has the opposite sign of the weighted Ricci curvature tensor of $\left(\mathbb{B}_{n}, v_{\alpha}\right)$, that is, the function above is always non-negative when $\alpha \leq 0$, and always negative when $\alpha>0$.

\section{REFERENCES}

[1] R. Aulaskari and H. Chen, Area inequality and $Q_{p}$ norm, J. Funct. Anal. 221 (2005), 1-24. MR.2124895 (2005k:30066)

[2] D. Bakry and M. Emery, Diffusions Hypercontractives, Lecture Notes in Math. 1123, Springer, Berlin, 1985, 117-206. MR0889476 (88j:60131)

[3] M. Beals, C. Fefferman and, R. Grossman, Strictly pseudoconvex domains in $\mathbb{C}^{n}$, Bull. Amer. Math. Soc. (N.S.) 8 (1983), 125-322. MR684898 (85a:32025)

[4] J. Burbea, Sharp inequalities for holomorphic functions, Illinois J. Math. 31 (1987), 248-264. MR882113 (89e:32012)

[5] R.B. Burckel, D.E. Marshall, D. Minda, P. Poggi-Corradini, and T.J. Ransford, Area, capacity and diameter version of Schwarz's lemma, Conformal Geom. Dynamics 12 (2008), 133-152. MR 2434356 (2010j:30050)

[6] T. Carleman, Zur Theorie der Minimalflächen, Math. Z. 9 (1921), 154-160. MR.1544458

[7] P. Duren, Theory of $H^{p}$ Spaces, Academic Press, New York, 1970. MR0268655 (42:3552)

[8] S. Fu and B. Wong, On strictly pseudoconvex domains with Kähler-Einsten Bergman metrics, Math. Res. Letters 4 (1997), 697-703. MR.1484700 (98m:32022)

[9] R.E. Greene and H. Wu, Curvature and complex analysis, Bull. Amer. Math. Soc. 77 (1971), 1045-1049. MR0283240(44:473)

[10] G.H. Hardy, The mean value of the modulus of an analytic function, Proc. London Math. Soc. 14 (1914), 269-277.

[11] H. Hedenmalm, private communication.

[12] M. Mateljević and M. Pavlović, New proofs of the isoperimetric inequality and some generalizations, J. Math. Anal. Appl. 98 (1984), 25-30. MR728515 (85c:30001)

[13] F. Morgan, Manifolds with density, Notices of the AMS, September 2005, 853-858. MR2161354 (2006g:53044)

[14] R. Osserman, A new variant of the Schwarz-Pick-Ahlfors lemma, Manusc. Math. 100 (1999), 123-129. MR1721627 (2000k:53035)

[15] Ch. Pommerenke, Boundary Behaviour of Conformal Maps, Springer-Verlag, BerlinHeidelberg, 1992. MR.1217706 (95b:30008)

[16] Z. Qian, Estimates for weighted volumes and applications, Quart. J. Math. Oxford (2) 48 (1997), 235-242. MR1458581 (98e:53058)

[17] K. Strebel, Quadratic Differentials, Series of Modern Surveys in Math., Band 5, SpringerVerlag, Berlin-Heidelberg, 1984. MR743423 (86a:30072)

[18] A.E. Taylor, New proofs of some theorems of Hardy by Banach space methods, Math. Magazine 23 (1950), 115-124. MR0033878(11:507a)

[19] D. Vukotić, A sharp estimate for $A_{\alpha}^{p}$ functions in $\mathbf{C}^{n}$, Proc. Amer. Math. Soc. 117 (1993), 753-756. MR.1120512 (93d:46042)

[20] D. Vukotić, The isoperimetric inequality and a theorem of Hardy and Littlewood, Amer. Math. Monthly 110 (2003), 532-536. MR.1984405

[21] J. Xiao, Geometric $Q_{p}$ Functions, Frontiers in Mathematics, Birkhäuser Verlag, Basel, 2006. MR2257688 (2008f:30003)

[22] K. Zhu, Spaces of Holomorphic Functions in the Unit Ball, Springer-Verlag, New York, 2005. MR2115155 (2006d:46035)

[23] K. Zhu, Translating inequalities between Hardy and Bergman spaces, Amer. Math. Monthly 111 (2004), 520-525. MR2076585 (2005d:46055)

Department of Mathematics and Statistics, Memorial University of Newfoundland, St. John's, NL A1C 5S7, Canada

E-mail address: jxiao@mun.ca

Department of Mathematics and Statistics, State University of New York, Albany, NEW YORK 12222

E-mail address: kzhu@albany.edu 